\title{
Transcriptome sequencing of olfactory related genes in olfactory transduction of large yellow croaker (Larimichthy crocea) in response to bile salts
}

\author{
Jiabao Hu ${ }^{1,2,3}$, Yajun Wang Corresp., 1,2,3 , Qijun Le ${ }^{1,2,3,4}$, Na Yu 1,2,3 , Xiaohuan Cao ${ }^{1,2,3}$ ， Siwen Kuang ${ }^{1,2,3}$, Man

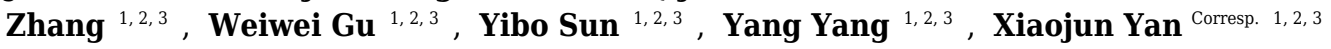 \\ ${ }^{1}$ Key Laboratory of Applied Marine Biotechnology, Ningbo University, Ministry of Education, Ningbo, China \\ 2 Key Laboratory of Marine Biotechnology of Zhejiang Province, Ningbo University, Ningbo, China \\ 3 College of Marine Sciences, Ningbo University, Ningbo, China \\ 4 Ningbo Entry-Exit Inspection and Quarantine Bureau Technical Centre, Ningbo, China \\ Corresponding Authors: Yajun Wang, Xiaojun Yan \\ Email address: wangyajun@nbu.edu.cn, yanxiaojun@nbu.edu.cn
}

Fish produce and release bile salts as chemical signalling substances that act as sensitive olfactory stimuli. To investigate how bile salts affect olfactory signal transduction in large yellow croaker (Larimichthy crocea), deep sequencing of olfactory epithelium was conducted to analyse olfactory-related genes in olfactory transduction. Sodium cholates (SAS) have typical bile salt chemical structures, hence we used four different concentrations of SAS to stimulate $L$. crocea, and the fish displayed a significant behavioural preference for $0.30 \%$ SAS. We then sequenced olfactory epithelium tissue, and identified 9938 unigenes that were significantly differentially expressed between SASstimulated and control groups, including 9055 up-regulated and 883 down-regulated unigenes. Subsequent Gene Ontology (GO) and Kyoto Encyclopedia of Genes and Genomes (KEGG) analyses found eight categories linked to olfactory transduction pathways that were highly enriched with some differentially expressed genes (DEGs), including Olfactory receptor (OR), Adenylate cyclase type $3(A D C Y 3)$ and Calmodulin $(C A L M)$. Genes in these categories were analysed by RT-qPCR, which revealed aspects of the pathway transformation between odor detection, and recovery and adaptation. The results provide new insight into the effects of bile salt stimulation in olfactory molecular mechanisms in fishes, and expands our knowledge of olfactory transduction, and signal generation and decline. 
1 Transcriptome sequencing of olfactory related genes in olfactory transduction

2 of large yellow croaker (Larimichthy crocea) in response to bile salts

4 Jiabao $\mathrm{Hu}^{1,2,3}$, Yajun Wang 1,2,3 *, Qijun Le 1,2,3,4, $\mathrm{Na} \mathrm{Yu}^{1,2,3}$, Xiaohuan Cao 1,2,3, Siwen Kuang 1, 2, 3, Man

5 Zhang ${ }^{1,2,3}$, Weiwei Gu ${ }^{1,2,3}$, Yibo Sun 1,2,3, Yang Yang 1,2,3, Xiaojun Yan 1, 2, 3 *

6

$7 \quad{ }^{1}$ Key Laboratory of Applied Marine Biotechnology, Ningbo University, Ministry of Education, Ningbo, China

$8 \quad{ }^{2}$ Key Laboratory of Marine Biotechnology of Zhejiang Province, Ningbo University, Ningbo, China

$9 \quad{ }^{3}$ College of Marine Sciences, Ningbo University, Ningbo, China

$10 \quad{ }^{4}$ Ningbo Entry-Exit Inspection and Quarantine Bureau Technical Centre, Ningbo, China

Correspondence:

Dr Yajun Wang,

14 wangyajun@nbu.edu.cn;

Dr Xiaojun Yan,

\section{Abstract}


Fish produce and release bile salts as chemical signalling substances that act as sensitive olfactory stimuli. To investigate how bile salts affect olfactory signal transduction in large yellow croaker (Larimichthy crocea), deep sequencing of olfactory epithelium was conducted to analyse olfactory-related genes in olfactory transduction. Sodium cholates (SAS) have typical bile salt chemical structures, hence we used four different concentrations of SAS to stimulate $L$. crocea, and the fish displayed a significant behavioural preference for $0.30 \%$ SAS. We then sequenced olfactory epithelium tissue, and identified 9938 unigenes that were significantly differentially expressed between SAS-stimulated and control groups, including 9055 up-regulated and 883 down-regulated unigenes. Subsequent Gene Ontology (GO) and Kyoto Encyclopedia of Genes and Genomes (KEGG) analyses found eight categories linked to olfactory transduction pathways that were highly enriched with some differentially expressed genes (DEGs), including Olfactory receptor (OR), Adenylate cyclase type $3(A D C Y 3)$ and Calmodulin $(C A L M)$. Genes in these categories were analysed by RT-qPCR, which revealed aspects of the pathway transformation between odor detection, and recovery and adaptation. The results provide new insight into the effects of bile salt stimulation in olfactory molecular mechanisms in fishes, and expands our knowledge of olfactory transduction, and signal generation and decline.

\section{Introduction}

Bile salts are highly structurally variable in vertebrates, and can be classified into three types; C (27) bile alcohols, C (27) bile acids, and C (24) bile acids, with default hydroxylation at C-3 and C-7 (Hofmann et al., 2010). They are biliary constituents derived from cholesterol that are synthesised in the liver and stored in the gall bladder (Haslewood, 1967). Regulated by different transport proteins, these salts are released into the intestinal lumen through enterohepatic circulation (Trauner and Boyer, 2003). Because bile salts can help intestines to digest and absorb dietary lipids and fat-soluble vitamins (Haslewood, 1967; Fuentes et al., 2018), they are included in the diet of fishes to improve growth and digestive enzyme activities (Deshimaru et al., 1982; Alam et al., 2015). Moreover, many studies on behaviour and physiology have reported that bile salts are important chemical signalling substances as well as effective olfactory stimuli in fishes, which have distinct sensitivity to different components (Zhang et al., 2001; Døving et al., 1980), but molecular studies have been limited. 
During olfactory activity, odourant molecules released into the environment bind to olfactory related receptors (Kaupp, 2010). To date, three types of receptor genes have been identified in fishes, namely olfactory receptors (ORs) (Freitag et al., 1998), vomeronasal receptors (VRs) (Freitag et al., 1995), and trace amineassociated receptors (TAARs) (Eisthen, 2004). Among them, OR genes play an essential role in many odor detecting activities (Hu et al., 2017; Yabuki et al., 2016; Bird et al., 2018). ORs encoding G protein-coupled receptors expressed in ciliated sensory neurons were previously identified in Rattus norvegicus (Buck and Axel, 1991). According to function, OR genes can be divided into two classes; class I ( $\alpha, \beta, \gamma, \delta, \varepsilon$ and $\zeta)$ and class II $(\eta, \theta, \kappa$ and $\lambda$ ) (Niimura and Nei, 2005; Hoover, 2013). In fishes, OR genes mainly belong to class I, which are believed to recognize water-soluble odours (Freitag et al., 1998; Zhou et al., 2011). ORs are member of a multigene family of G protein-coupled receptors and seven transmembrane domain proteins (Buck and Axel, 1991), and $\mathrm{G}_{\text {oolf }}$, one subunit of the $\mathrm{G}$ protein-coupled to $\mathrm{OR}$, activates adenylyl cyclase in olfactory sensory cells (Hansen et al., 2004; Schild and Restrepo, 1998; Gonçalves et al., 2016). Olfactory signals are eventually transmitted to the brain via regulation of distinct factors in olfactory transduction (Meredith et al., 2012).

Many recent studies have focused on the identification and expression of olfactory related receptor genes in fishes (Zhu et al., 2017; Fatsini et al., 2016; Cui et al., 2017). In the present study, to increase our knowledge of gene expression in the whole olfactory transduction system in fishes following stimulation by bile salts, we identified the optimal concentration for stimulation in L. crocea, and performed deep sequencing of olfactory epithelium tissue using an Illumina HiSeq 2500 platform. Subsequent GO and KEGG pathway analyses identified significantly differentially expressed genes (DEGs) enriched in eight categories related to olfactory transduction, and gene expression levels were confirmed for selected genes by RT-qPCR. The results indicate that bile salts have attractant effects on L. crocea. The findings provide new insight into effects of bile salt stimulation on olfactory molecular mechanisms in fishes, and expand our knowledge of olfactory transduction and olfactory signalling.

\section{Methodology}

\section{Fish and bile salt stimulation treatments}


The large yellow croakers (mean weight $=20 \pm 1.2 \mathrm{~g}$ ) used in the study were commercially reared at 25$27^{\circ} \mathrm{C}$ in Xiangshan Bay, Zhejiang, China. All fish experiments were conducted in accordance with the recommendations in the National Institutes of Health Guide for the Care and Use of Laboratory Animals. The Animal Care and Use Committee of Ningbo University approved the protocols.

Sodium cholates (SAS) with typical bile salt chemical structures were chosen for stimulation treatments (Haslewood, 1967). Four different concentrations of SAS diluted in distilled water $(0.20 \%, 0.30 \%, 0.40 \%$ and $0.50 \%$ ) were applied to SAS groups, while the control group (Control) was treated with by distilled water alone. SAS was released into cultured water slowly using an air stone tube (submerged in the center of the pond) equipped with a $20 \mathrm{~mL}$ syringe (100 individuals per group, three independent biological replications). The behavioural responses of each fish were classified as two types: biting the air stone (a positive feeding response), and swimming close to the air stone without biting (a positive movement response). We replaced the tested fish with another new fish for subsequent replications at all concentrations. Culture water was changed after every test, and each test was performed at $24 \mathrm{~h}$ intervals. Behaviours were recorded with a camera for 5 min, and the number of each type of response was recorded and analysed statistically by one-way analysis of variance (ANOVA) and Tukey’s multiple comparison tests (SPSS, version 16.0).

The concentration that produced the highest number of behavioural responses was used for subsequent stimulation experiments, which were performed as described as above. After stimulation, we captured control group fish, and fish from SAS groups exhibiting significantly positive feeding responses, and immediately extracted olfactory epithelium tissues by cutting the nostrils. Olfactory epithelium tissues from 15 randomly selected fish were extracted and pooled into three $1.5 \mathrm{~mL}$ RNAase-free tubes (three independent biological replicates for each group) and stored in liquid nitrogen for RNA-seq and RT-qPCR experiments.

\section{RNA isolation, library construction and Illumina sequencing}

Total RNAs were extracted using TRIzol reagent (Invitrogen, CA, USA). RNA was monitored on 1\% agarose gels, RNA purity was checked using a NanoPhotometer spectrophotometer (IMPLEN, CA, USA), RNA concentration was measured using a Qubit RNA Assay Kit with a Qubit 2.0 Fluorimeter (Life Technologies, CA, USA), and RNA integrity was assessed using an RNA Nano 6000 Assay Kit with a 
Bioanalyzer 2100 system (Agilent Technologies, CA, USA).

Sequencing libraries were generated using an NEBNext Ultra RNA Library Prep Kit for Illumina (NEB, CA, USA) and barcodes were added to attribute sequences to each sample. Clustering of the barcoded samples was performed on a cBot Cluster Generation System using a TruSeq PE Cluster Kit v3-cBot-HS (Illumina). After cluster generation, library preparations were sequenced on an Illumina HiSeq 2500 platform and pairedend reads were generated.

\section{Assembly of sequencing data and gene annotation}

Raw data were firstly processed through in-house perl scripts, and clean data were obtained by removing reads containing adapters or poly-N sequences, and reads of low quality. Q20, Q30 and GC values were calculated, and all downstream analyses were based on high-quality clean data.

The reference genome of the large yellow croaker was downloaded from the National Center of Genome Research website (https://www.ncbi.nlm.nih.gov/genome/?term=JPYK-00000000) (Ao et al., 2015), and data were mapped using TopHat (version 2.0.12) and Bowtie2 (Trapnell et al., 2009; Langmead et al., 2009). Unigenes were searched using BLASTX against the National Center for Biotechnology Information (NCBI) non-redundant protein sequence (NR) database, the NCBI non-redundant nucleotide sequence (NT) database, and Gene Ontology (GO), KEGG Orthology (KO) and SwissProt databases with an E-value threshold of $10^{-5}$.

\section{Identification of differentially expressed genes (DEGs) and functional analysis}

Differential expression analysis was performed using the DEGSeq R package (1.20.0) and Reads per Kilobase Millon Mapped Reads (RPKM) values (Mortazavi et al., 2008). The resulting $p$-values were adjusted using the Benjamini and Hochberg's approach for controlling the false discovery rate. DEGs were selected with the criteria adjusted $p$-value $<0.05$ and $\mid \log 2$ fold-change $\mid>1$.

GO enrichment analysis of DEGs was implemented by the GOseq R package, and KEGG enrichment was used to identify putative functions and pathways of DEGs (http://www.genome.jp/kegg/).

\section{Real-time quantitative PCR (RT-qPCR) analysis}


Total RNA was reverse-transcribed into cDNA using a PrimeScript RT Reagent Kit (TaKaRa, Dalian, China). Primers were designed using Primer 5.0 software (Table 1). $\beta$-actin served as an internal normalisation control for RT-qPCR analysis, and reactions contained $2 \mu \mathrm{l}$ cDNA, $1 \mu \mathrm{l}$ forward and reverse primers, $10 \mu \mathrm{l}$ SYBR Green I Master Mix (TaKaRa), and $6 \mu l$ water. RT-qPCR was performed on an Eppendorf PCR machine (Mastercycler ep Realplex, Hamburg, Germany) with one cycle at $95^{\circ} \mathrm{C}$ for $2 \mathrm{~min}$, followed by 40 cycles at $95^{\circ} \mathrm{C}$ for $15 \mathrm{~s}, 58^{\circ} \mathrm{C}$ for 15 $\mathrm{s}$, and $72^{\circ} \mathrm{C}$ for $20 \mathrm{~s}$. The relative expression level was calculated using the $2^{-\Delta \Delta \mathrm{CT}}$ method, and statistical analysis was performed using independent sample t-tests (SPSS, version 16.0).

\section{Results}

\section{Selecting the optimal concentration of bile salts and assessing fish responses}

For bile salt stimulation treatments, SAS was diluted four different concentrations, added slowly to water, and L. crocea responses were monitored (Fig. 1). For feeding responses, the fish reacted most obviously to $0.30 \%$ SAS $(17.67 \pm 0.58$ fish responded in $5 \mathrm{~min})$. Meanwhile, for movement responses, they exhibited optimal attraction responses to $0.30 \%$ SAS $(64.33 \pm 3.51$ fish responded in $5 \mathrm{~min})$ and $0.40 \%(48.33 \pm 3.51$ fish responded in $5 \mathrm{~min}$ ). Thus, we chose $0.30 \% \mathrm{SAS}$ for subsequent RNA-seq and RT-qPCR experiments.

\section{Results and analysis of transcriptome sequencing data}

cDNA libraries were constructed from control and SAS groups, resulting in 39,805,502 and 39,116,990 raw reads, and more than $81 \%$ raw reads were filtered to yield clean reads. In total, 25,684,902 and 25,830,011 clean reads were mapped to the reference genome of $L$. crocea for control and SAS groups, respectively, and the Q30 value was $>95 \%$ for libraries (Table 2 ).

\section{Identification and functional annotation of DEGs}

Transcriptome data from olfactory epithelium tissue of control and SAS groups were compared, and 19,197 unigenes were annotated, of which 9938 DEGs met the criteria $(\mid \log 2$ Foldchange $\mid>1$ and $p<0.05)$. Of these, 9055 were up-regulated and 883 were down-regulated (Fig. 2). Three types of olfactory-related receptor genes 
162 were found to be differentially expressed in our data (all up-regulated), comprising 59 ORs, two VRs and 17 163 TAARs.

164 To investigate the functions of DEGs, 9245 unigenes (8424 up-regulated and 821 down-regulated) were assessed 165 in terms of the three main GO classifications, namely biological processes (BP), cellular component (CC), and 166 molecular function (MF; Fig. 3). According to the criteria ( $p$-value $<0.001$ ), single-organism process 167 (GO:0044699), intrinsic to membrane (GO:0031224) and substrate-specific channel activity (GO:0022838) were highly represented, and potentially play an important role in olfactory responses to bile salts.

To identify KEGG pathways between control and SAS groups, 3140 DEGs were mapped to 321 pathways, and 20 pathways were highly enriched according to $q$-value $<0.05$ (Table 3 ). Among these pathways, olfactory transduction (map04740) was mainly involved in olfactory responses to bile salts. In this pathway, 73 differentially expressed olfactory-related genes were enriched among eight categories including calmodulin (CALM, k02183), adenylate cyclase 3 (ADCY3, k08043), guanine nucleotide-binding protein G (olf) subunit alpha (GNAL, k04633), calcium/calmodulin-dependent protein kinase (CaM kinase) II (CAMK2, k04515), olfactory receptor (OLFR, k04257), cyclic nucleotide gated channel beta 1 (CNGB1, k04952), cyclic nucleotide gated channel alpha 3 (CNGA3, k04950) and protein kinase A (PKA, k04345; Fig. 4). Significantly differentially expressed olfactory-related genes in these categories were subsequently analysed RT-qPCR (Table 4).

In the olfactory transduction pathway (Fig. 4), olfactory stimulation could be divided into odor detection, and recovery and adaptation. During odor detection, odour-activated OLFR stimulates G protein release protein GNAL, and ADCY3, which is positively regulated by GNAL, stimulates cAMP release; cAMP is then transported out of the olfactory cell, and $\mathrm{Na}^{+}$and $\mathrm{Ca}^{2+}$ are exchanged by CNGB1. This process is an example of signal production and amplification. During recovery and adaptation, an increase in cAMP activates PKA, which phosphorylates OLFR; meanwhile, CALM represses CNGB1 and activated CAMK2 to suppress ADCY 3 by phosphorylation. This process represents an example of signal suppression.

\section{RT-qPCR analysis of eight categories related to olfactory transduction}

The expression levels of nine DEGs related to the olfactory transduction pathway were validated by RT-qPCR. 
These genes were all significantly expressed in the olfactory epithelium $\left(* p<0.05\right.$ and $\left.{ }^{* *} p<0.01\right)$, especially $C A M K 2, A D C Y 3, O R 2 D 3$ and $C N G B 1$, confirming the reliability of the transcriptome sequencing data (Fig. 5). Furthermore, $C N G B 1 a$ and $C N G B 1 \quad b$ both belonging to $C N G B 1$, and $C N G B 1$ a (up-regulated) displayed more significant differential expression than $C N G B 1 b$ (down-regulated).

\section{Discussion}

\section{Bile salts act as effective olfactory stimuli in fishes}

Fish can display different olfactory responses to different levels of odours. Using electroolfactograms (EOGs), many fish species have been shown to possess specific thresholds to different levels of cholic acid (CA) (Meredith et al., 2012; Døving et al., 1980). In the present study, we found that L. crocea acted differently to different concentrations of bile salts based on behavioural analysis, and responded optimally to $0.3 \%$ SAS rather than to higher levels. We believe that fishes have limited olfactory related receptors, which leads to limited olfactory ability, explaining why they do not exhibit significantly more intense behaviour with increased levels of odours. This suggests that fishes may possess a maximum detection peak for concentrations of certain stimuli, and a similar phenomenon has been observed in other studies (Zhao, 2007; Hu et al., 2017).

The olfactory sensitivity of fishes can also be measured using EOGs. However, unlike EOG analysis, our behavioural experiments revealed fish response to stimuli directly (approach and avoidance). By imitating conditions in which fishes detect bile salts in natural environments, we found that $L$. crocea performed feeding movements upon exposure to SAS, which suggests that the fish had a particular preference toward SAS. Indeed, many studies have demonstrated that some bile salts could be good phagostimulants for fish feed (Hu et al., 2017; Rolen and Caprio, 2008; Yamashita et al., 2010), suggesting that they may act on both olfactory and taste pathways in fish, and might be good attractants.

\section{Analysis of olfactory related receptor genes in the odorant transduction cascade}

In L. crocea, OR gene family is the largest one of three receptor genes families (Ao et al., 2015; Zhou et al., 2011). In our current study, 59 OR genes were found to be all up-regulated after the fish were stimulated, many more than two other two types of receptors, consistent with previous studies (Saraiva and Korsching, 
216

217

218

219

220

221

222

223

224

225

226

227

228

229

230

231

232

233

234

235

236

237

238

239

240

241

2007; Hashiguchi and Nishida, 2006; Hu et al., 2017). Thus, ORs appear to be the major receptors responding

to bile salts in L. crocea. Fish produce and release bile salts as sex pheromones to communicate with other individuals (Zhang et al., 2001). However, regarding pheromone receptors in the epithelium (Muramoto et al., 2011), only two VR genes were differentially expressed (up-regulated) in the present study. We speculate that the fish used in our study might be juveniles, hence VRs were not sensitive to sex pheromones at this stage of the life cycle. Moreover, 17 TAAR genes were found to be all up-regulated following stimulation by SAS in our study. Interestingly, TAARs could only be activated by amines at trace level in a previous study (Borowsky et al., 2001), and SAS is not an amine, suggesting that the fish might release some amines substances to communicate with each other in response to SAS. Our study reveals that ORs might be the main bile salt receptors in the olfactory epithelium during different developmental stages in fish species.

\section{Signal transduction and regulation components}

During signal transduction, ORs bind to their corresponding G proteins, among which $\mathrm{G} \alpha$ is one of most important subunits (Jones and Reed, 1989). In the present study, $\mathrm{G}_{\text {oolf }}$ was released in the olfactory transduction pathway after ORs were activated by SAS, and two $\mathrm{G}_{\alpha \text { olf }}$ genes were up-regulated alongside high expression of $O R s$, which suggests that olfactory receptors bind to $\mathrm{G}$ protein possessing the $\mathrm{G}_{\text {oolf }}$ subunit. Some studies on olfactory sensory neurons also have confirmed similar binding relationships of them (Jones and Reed, 1989; Ronnett and Moon, 2002). However, only three $\mathrm{G}_{\text {oolf }}$ genes were identified in L. crocea, indicating that they may be a small gene family in this fish species.

In the present study, the $\mathrm{G}_{\text {oolf }}$ subunits activated ADCY3, which led to a rise in cAMP levels during olfactory transduction, which is of clear relevance to signal transduction (Jones and Reed, 1989; Dhallan et al., 1990; Menco et al., 1992). We also found that ADCY3 was enriched among up-regulated genes in the pathway, which suggests that this factor could act positively on signal transduction, and play a key role in regulating transformation of the pathway via the cAMP levels. Moreover, ADCY3 was the first factor in secondary signal transduction (Fig. 4), and some other studies have reported that signal transduction can be disrupted if ADCY3 genes are mutated (Brunet et al., 1996; Hacker, 2000). Thus, our results indicate that 
242

243

245

246

247

ADCY3 is one of most important factors mediating signal transduction between primary and secondary signal transduction.

In odor detection of olfactory transduction, high cAMP levels produced by ADCY3 activated CNGB1, leading to the entrance of $\mathrm{Na}^{+}$and $\mathrm{Ca}^{2+}$ into olfactory sensory cells. Other studies have reported similar results (Michalakis et al., 2006; Kaupp and Seifert, 2002). Thus, we speculate that an increase in these two ions by CNGB1 might appeared to suppress the expression of $C N G A 3$, another same functional channel proteinencoding genes, due to competition effects. In recovery and adaptation of the pathway, activated CALM resulting from increased $\mathrm{Ca}^{2+}$ regulated by CAMK2 suppresses CNGB1, leading to a drop in cAMP level indirectly, consistent with observations in previous studies (Cheung, 1980; Lynch and Barry, 1989; Menini et al., 1995). Thus, a series of interactions may cause $\mathrm{Ca}^{2+}$ levels to decline, reducing the intracellular and extracellular charge difference. In addition, $C N G B 1 a$ was expressed at higher levels than $C N G B 1 b$ based on RT-qPCR, which indicates that signal transduction in fish might transform odor detection into recovery and adaptation. These findings reveal that $C N G B 1 a$ and $C N G B 1 b$ may be involved in signal production and decline in the pathway, respectively.

PKA can help ORs to bind to G proteins (Daaka et al., 1997; Zamah et al., 2002) in a mechanism mediated by cAMP (Chang et al., 2006), and our results showed that PKA (up-regulated) was enriched during the recovery and adaptation aspect of olfactory transduction. Thus, PKA might suppress the initial signal level by hindering the separation between receptor and G protein by phosphorylation. Combined with the results of a previous study (Taiwo et al., 1989), our findings indicate that the inhibitory action of PKA may be activated through a change in cAMP concentration due to binding between ORs and G proteins, and indirectly by suppression of ADCY3. These factors might alter the electric charge in olfactory sensory cells by meditating the ion concentration, which might lead to changes in electric signalling between olfactory receptor cells and olfactory sensory neurons, consistent with some previous reports (Menini et al., 1995; Lynch and Barry, 1989). Our results therefore indicate that odor detection in fish may occur quite rapidly, or a long time after, stimulation by bile salts, and recovery and adaptation may occur once fish become familiar to this stimulation.

\section{Conclusion}


269

270

271

272

273

274

275

276

277

278

279

280

281

282

283

284

285

286

287

In the present study, L. crocea displayed a significant behavioural preference for $0.3 \%$ SAS, which could be a good attractant in fishes. We performed transcriptome sequencing of olfactory epithelium tissue to identify olfactory-related genes involved in the olfactory transduction pathway, and eight categories were found to be highly enriched with DEGs in related DEGs, especially $C A M K 2, A D C Y 3, O R 2 D 3$ and $C N G B 1$. The pathway could be divided into two processes: odor detection, and recovery and adaptation, and involves DEGs such as $C A M K 2, C A L M, C N G B 1$ and $P K A$ that may regulate conversion between the two processes. Our results provide new insight into the effects of bile salt stimulation on olfactory molecular mechanisms in fishes, and expand our knowledge of olfactory transduction and signal production and decline.

\section{Acknowledgements}

We thank Shunshun Tao of the Xiangshan Harbor Aquaculture and Larva Limited Company and Dr. Bao of Wanli College of Zhejing.

\section{References}

Ao, J., Mu, Y., Xiang, L., Fan, D., Feng, M., Zhang, S., Shi, Q., Zhu, L., Li, T., and Ding, Y. (2015). Genome Sequencing of the Perciform Fish Larimichthys crocea Provides Insights into Molecular and Genetic Mechanisms of Stress Adaptation. Plos genetics 11, 8-8. DOI: 10.1371/journal.pgen. 1005118

Alam, M. S., Teshima, S., Ishikawa, M., and Koshio, S. (2015). Effects of ursodeoxycholic acid on growth and digestive enzyme activities of Japanese flounder Paralichthys olivaceus (Temminck \& Schlegel). Aquaculture Research 32, 235-243. DOI: 10.1046/j.1355-557x.2001.00020.x

BIRD, D. J., MURPHY, W. J., FOXROSALES, L., HAMID, I., EAGLE, R. A. \& VAN, B. V. 2018. Olfaction written in bone: cribriform plate size parallels olfactory receptor gene repertoires in Mammalia. Proceedings of the Royal Society B Biological Sciences, 285, 20180100. DOI: $10.1098 /$ rspb.2018.0100

Brunet, L. J., Gold, G. H., and Ngai, J. (1996). General Anosmia Caused by a Targeted Disruption of the Mouse Olfactory Cyclic Nucleotide-Gated Cation Channel. Neuron 17, 681-693. DOI: 10.1016/S08966273(00)80200-7 
296

297

298

299

300

301

302

303

304

305

306

307

308

309

310

311

312

313

314

Borowsky, B., Adham, N., Jones, K. A., Raddatz, R., Artymyshyn, R., Ogozalek, K. L., Pathirana, S. (2001). Trace amines: identification of a family of mammalian G protein-coupled receptors. Proceedings of the National Academy of Sciences of the United States of America, 98(16), 8966-8971. doi: $10.1073 /$ pnas. 151105198

Buck, L., and Axel, R. (1991). A novel multigene family may encode odorant receptors: a molecular basis for odor recognition. Cell 65, 175-187. DOI: 10.1016/0092-8674(91)90418-X

Chang, L. Y., Yu-Ming, X. U., and Zhang, S. M. (2006). cAMP-PKA signal pathway mediates the promotive effect of NGF on axon regeneration in cerebral ischemia-reperfusion rats. Chinese Journal of Geriatric Heart Brain \& Vessel Diseases.

Cheung, W. Y. (1980). Calmodulin plays a pivotal role in cellular regulation. Science 207, 19-27. DOI: $10.1126 /$ science. 6243188

CUI, R., DELClOS, P. J., SCHUMER, M. \& ROSENTHAL, G. G. 2017. Early social learning triggers neurogenomic expression changes in a swordtail fish. Proceedings of the Royal Society B Biological Sciences. DOI: 10.1098/rspb.2017.0701

Døving, K. B., Selset, R., and Thommesen, G. (1980). Olfactory sensitivity to bile acids in salmonid fishes. Acta Physiologica Scandinavica 108, 123\&ndash;131. DOI:10.1111/j.1748-1716.1980.tb06509.x

Daaka, Y., Luttrell, L. M., and Lefkowitz, R. J. (1997). Switching of the coupling of the beta2-adrenergic receptor to different $\mathrm{G}$ proteins by protein kinase A. Nature 390, 88-91. DOI: 10.1038/36362

Deshimaru, O., Kuroki, K., and Yone, Y. (1982). Suitable levels of lipids and ursodesoxycholic acid in diet for yellowtail [Fish]. Bulletin of the Japanese Society of Scientific Fisheries. DOI: 10.2331/suisan.48.1265

Dhallan, R. S., Yau, K. W., Schrader, K. A., and Reed, R. R. (1990). Primary structure and functional expression of a cyclic nucleotide-activated channel from olfactory neurons. Nature 347, 184-7. DOI: $10.1038 / 347184 \mathrm{a} 0$

Eisthen, H. L. (2004). The goldfish knows: olfactory receptor cell morphology predicts receptor gene expression. Journal of Comparative Neurology 477, 341-346. DOI: 10.1002/cne.20258 
321

322

323

324

325

326

327

328

329

330

331

332

333

334

335

336

337

338

339

340

341

342

343

344

345

346

347

FATSINI, E., BAUTISTA, R., MANCHADO, M. \& DUNCAN, N. J. 2016. Transcriptomic profiles of the upper olfactory rosette in cultured and wild Senegalese sole ( Solea senegalensis ) males. Comp Biochem Physiol Part D Genomics Proteomics, 20, 125-135. DOI: 10.1016/j.cbd.2016.09.001

Freitag, J., Krieger, J., Strotmann, J., and Breer, H. (1995). Two classes of olfactory receptors in xenopus laevis. Neuron 15, 1383-92. DOI: 10.1016/0896-6273(95)90016-0

Freitag, J., Ludwig, G., Andreini, I., Rössler, P., and Breer, H. (1998). Olfactory receptors in aquatic and terrestrial vertebrates. Journal of Comparative Physiology A 183, 635-650. DOI: 10.1007/s003590050287

FUENTES, J., RIBEIRO, L. \& ARAG O, C. 2018. Bile salts regulate ion transport in the intestine of Senegalese sole. Aquaculture, 495, 842-848. DOI: 10.1016/j.aquaculture.2018.06.050

GON ALVES, I., HUBBARD, P. C., TOM S, J., QUINTELA, T., TAVARES, G., CARIA, S., BARREIROS, D. \& SANTOS, C. R. 2016a. 'Smelling'the cerebrospinal fluid: olfactory signaling molecules are expressed in and mediate chemosensory signaling from the choroid plexus. The FEBS journal, 283, 17481766. DOI: $10.1111 /$ febs. 13700

Hacker, B. (2000). Disruption of the type III adenylyl cyclase gene leads to peripheral and behavioral anosmia in transgenic mice. Neuron 27, 487-97. DOI: 10.1016/S0896-6273(00)00060-X

Hansen, A., Anderson, K. T., and Finger, T. E. (2004). Differential distribution of olfactory receptor neurons in goldfish: structural and molecular correlates. Journal of Comparative Neurology 477, 347. DOI: $10.1002 / \mathrm{cne} .20202$

Hashiguchi, Y., and Nishida, M. (2006). Evolution and origin of vomeronasal-type odorant receptor gene repertoire in fishes. BMC Evolutionary Biology 6, 1-13. DOI: 10.1186/1471-2148-6-76

Haslewood, G. A. (1967). Bile salt evolution. Journal of Lipid Research 8, 535-550.

Hofmann, A. F., Hagey, L. R., and Krasowski, M. D. (2010). Bile salts of vertebrates: structural variation and possible evolutionary significance. Journal of Lipid Research 51, 226-46. DOI: 10.1194/jlr.R000042

Hoover, K. C. (2013). "Evolution of Olfactory Receptors," Humana Press. DOI: 10.1007/978-1-62703-377$0 \_18$

HU, J., WANG, Y., LE, Q., NA, Y., CAO, X., ZHENG, H., KUANG, S., MAN, Z., ZHENG, J. \& WU, X. (2017). Transcriptomic analysis reveals olfactory-related genes expression in large yellow croaker 
348

349

350

351

352

353

354

355

356

357

358

359

360

361

362

363

364

365

366

367

368

369

370

371

372

373

374

(Larimichthys crocea) regulated by taurine: May be a good phagostimulant for all-plant protein diets. Aquaculture Research, 49. DOI: 10.1111/are.13559

Jones, D. T., and Reed, R. R. (1989). Golf: an olfactory neuron specific-G protein involved in odorant signal transduction. Science 244, 790-795. DOI: 10.1126/science.2499043

Kapiloff, M. S., Mathis, J. M., Nelson, C. A., Lin, C. R., and Rosenfeld, M. G. (1991). Calcium/calmodulindependent protein kinase mediates a pathway for transcriptional regulation. Proceedings of the National Academy of Sciences of the United States of America 88, 3710-4.

KAUPP, U. B. 2010. Olfactory signalling in vertebrates and insects: differences and commonalities. Nature Reviews Neuroscience, 11, 188-200.

Kaupp, U. B., and Seifert, R. (2002). Cyclic nucleotide-gated ion channels. Physiological Reviews 82, 769. DOI: $10.1152 /$ physrev.00008.2002

Langmead, B., Trapnell, C., Pop, M., and Salzberg, S. L. (2009). Ultrafast and memory-efficient alignment of short DNA sequences to the human genome. Genome Biology 10, 1-10.

Lynch, J. W., and Barry, P. H. (1989). Action potentials initiated by single channels opening in a small neuron (rat olfactory receptor). Biophysical Journal 55, 755-768. DOI: 10.1016/S0006-3495(89)82874-7

Menco, B. P. M., Bruch, R. C., Dau, B., Danho, and Waleed (1992). Ultrastructural localization of olfactory transduction components: the G protein subunit Gand type III adenylyl cyclase. Neuron 8, 441. DOI: 10.1016/0896-6273(92)90272-F

Meredith, T. L., Caprio, J., and Kajiura, S. M. (2012). Sensitivity and specificity of the olfactory epithelia of two elasmobranch species to bile salts. Journal of Experimental Biology 215, 2660-2667. DOI: $10.1242 /$ jeb.066241

Menini, A., Picco, C., and Firestein, S. (1995). Quantal-like current fluctuations induced by odorants in olfactory receptor cells. Nature 373, 435-437. DOI: $10.1038 / 373435 \mathrm{a} 0$

Michalakis, S., Reisert, J., Geiger, H., Wetzel, C., Zong, X., Bradley, J., Spehr, M., Hüttl, S., Gerstner, A., and Pfeifer, A. (2006). Loss of CNGB1 Protein Leads to Olfactory Dysfunction and Subciliary Cyclic Nucleotide-gated Channel Trapping. The Jounal of biological chemistry 281, 35156. DOI: 10.1074/jbc.M606409200 
375 Mortazavi, A., Williams, B. A., Mccue, K., Schaeffer, L., and Wold, B. (2008). Mapping and quantifying mammalian transcriptomes by RNA-Seq. Nature Methods 5, 621-628. DOI: 10.1038/nmeth.1226

Muramoto, K., Hagino-Yamagishi, K., Tonosaki, K., and Kaba, H. (2011). Accessory olfactory bulb neurons are required for maintenance but not induction of $\mathrm{V} 2 \mathrm{R}$ vomeronasal receptor gene expression in vitro. Neuroscience Letters 500, 6-9. DOI: 10.1016/j.neulet.2011.05.232

Niimura, Y., and Nei, M. (2005). Evolutionary dynamics of olfactory receptor genes in fishes and tetrapods. Proceedings of the National Academy of Sciences 102, 6039-44. DOI: 10.1073/pnas.0501922102

ROLEN, S. H. \& CAPRIO, J., . 2008. Bile salts are effective taste stimuli in channel catfish. Journal of Experimental Biology, 211, 2786-91. DOI: 10.1242/jeb.018648

Ronnett, G. V., and Moon, C. (2002). G proteins and olfactory signal transduction. Annual Review of Physiology 64, 189-222. DOI: 10.1146/annurev.physiol.64.082701.102219

Saraiva, L. R., and Korsching, S. I. (2007). A novel olfactory receptor gene family in teleost fish. Genome Research 17, 1448-57. DOI: 10.1101/gr.6553207

Schild, D., and Restrepo, D. (1998). Transduction mechanisms in vertebrate olfactory receptor cells. Physiological Reviews 78, 429-466. DOI: 10.1152/physrev.1998.78.2.429

Taiwo, Y. O., Bjerknes, L. K., Goetzl, E. J., and Levine, J. D. (1989). Mediation of primary afferent peripheral hyperalgesia by the cAMP second messenger system. Neuroscience 32, 577-80. DOI: 10.1016/03064522(89)90280-7

Trapnell, C., Pachter, L., and Salzberg, S. L. (2009). TopHat: discovering splice junctions with RNA-Seq. Bioinformatics 25, 1105-11. DOI:10.1093/bioinformatics/btp120

Trauner, M., and Boyer, J. L. (2003). Bile salt transporters: molecular characterization, function, and regulation. Physiological Reviews 83, 633-71. DOI: 10.1152/physrev.00027.2002

Wissinger, B., ., Gamer, D., ., JaGle, H., ., Giorda, R., ., Marx, T., ., Mayer, S., ., Tippmann, S., ., Broghammer, M., ., Jurklies, B., ., and Rosenberg, T., . (2001). CNGA3 mutations in hereditary cone photoreceptor disorders. American Journal of Human Genetics 69, 722-37. DOI: 10.1086/323613 
400

401

402

403

404

405

406

407

408

409

410

411

412

YABUKI, Y., KOIDE, T., MIYASAKA, N., WAKISAKA, N., MASUDA, M., OHKURA, M., NAKAI, J., TSUGE, K., TSUCHIYA, S. \& SUGIMOTO, Y. 2016. Olfactory receptor for prostaglandin F2[alpha] mediates male fish courtship behavior. Nature Neuroscience, 19, 897. DOI: 10.1038/nn.4314

YAMASHITA, S., YAMADA, T. \& HARA, T. J. 2010. Gustatory responses to feeding- and non-feedingstimulant chemicals, with an emphasis on amino acids, in rainbow trout. Journal of Fish Biology, 68, 783-800. DOI: 10.1111/j.0022-1112.2006.00965.x

Zamah, A. M., Delahunty, M., Luttrell, L. M., and Lefkowitz, R. J. (2002). Protein kinase A-mediated phosphorylation of the beta 2-adrenergic receptor regulates its coupling to Gs and Gi. Demonstration in a reconstituted system. Journal of Biological Chemistry 277, 31249-31256. DOI: 10.1074/jbc.M202753200

Zhang, C., Brown, S. B., and Hara, T. J. (2001). Biochemical and physiological evidence that bile acids produced and released by lake char (Salvelinus namaycush) function as chemical signals. Journal of Comparative Physiology B 171, 161. DOI: 10.1007/s003600000170

Zhao, H. Y. (2007). Studies on feeding stimulants for gibel carp(Carassius auratus gibelio). This is for Doctor of Science.Institute of Hydrobiology, Chinese Academy of Sciences, Wuhan.

Zhou, Y., Yan, X., Xu, S., Zhu, P., He, X., and Liu, J. (2011). Family structure and phylogenetic analysis of odorant receptor genes in the large yellow croaker (Larimichthys crocea). BMC Evolutionary Biology 11, 2546-2552. DOI: $10.1186 / 1471-2148-11-237$

ZHU, G., WANG, L., TANG, W., WANG, X. \& WANG, C. 2017. Identification of olfactory receptor genes in the Japanese grenadier anchovy Coilia nasus. Genes \& Genomics, 39, 521-532. DOI: $10.1007 / \mathrm{s} 13258-017-0517-8$ 
Figure 1

Fish responses to increasing concentrations of sodium cholates (SAS).

The number of fish displaying feeding (blue bars) or movement (orange bars) responses were quantified. Responses were considered significant $a * 0.01<p<0.05$ and ${ }^{* *} p<0.01$ levels compared with controls. Fish exhibited optimal behavioural performance in response to $0.3 \%$ SAS.

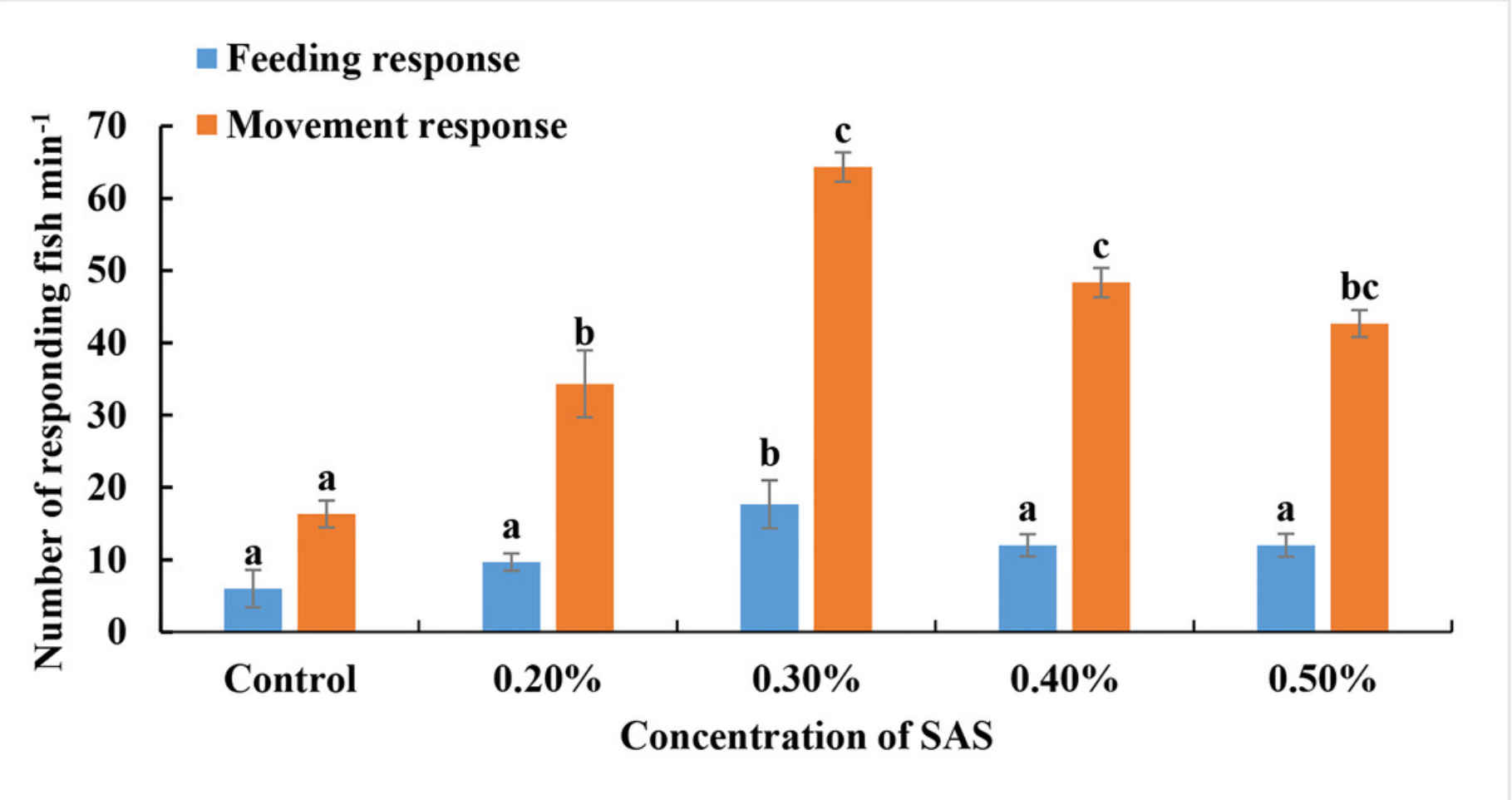


Figure 2

DEGs identified following bile salt stimulation.

Red spots represent up-regulated genes and green spots indicate down-regulated genes.

Grey spots represent genes showing no obvious change between control and SAS groups.

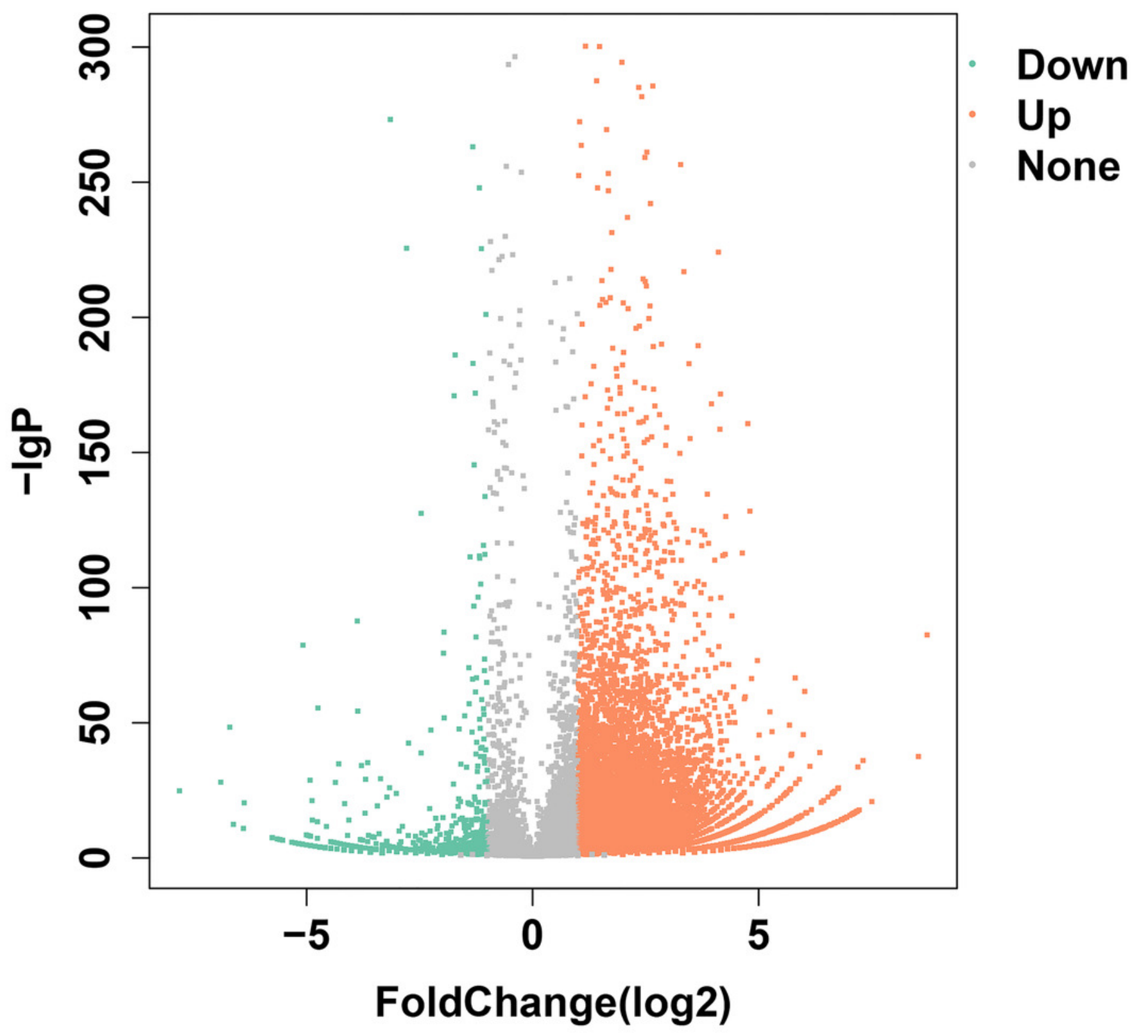


Figure 3

GO analysis of DEGs identified by comparing control and SAS groups. Orange represents up-regulated genes and green indicates down-regulated genes.

The height of bars is proportional to the number of DEGs.

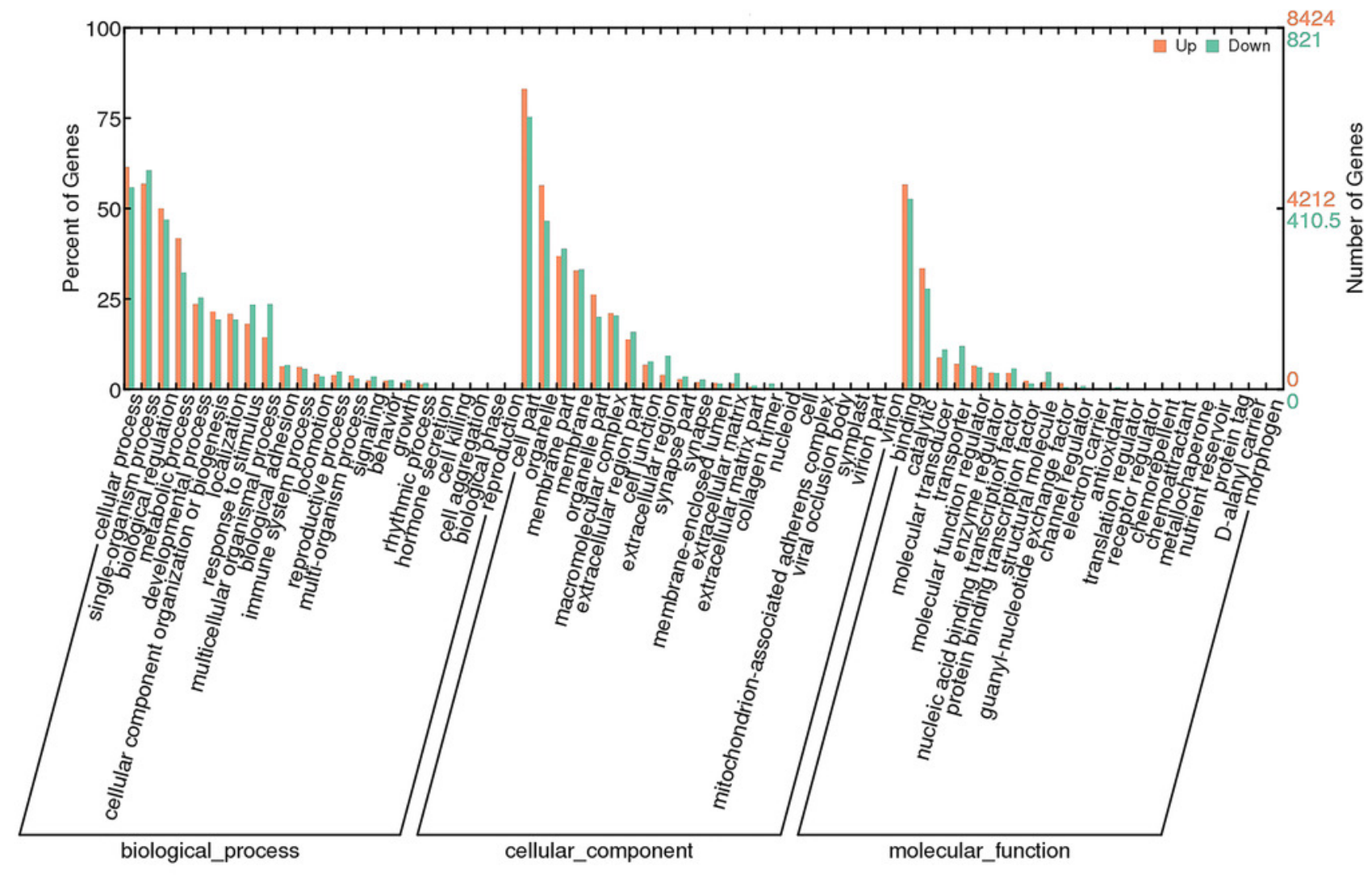


Figure 4

Significantly differentially expressed genes identified by KEGG in eight categories related to the olfactory transduction pathway.

Red indicates significantly up-regulated genes, green indicates significantly down-regulated genes, and blue indicates genes that were both up- and down-regulated. (Image credit: https://www.kegg.jp/kegg-bin/show_pathway?map04740)

\section{OLFACTORY TRANSDUCTION}
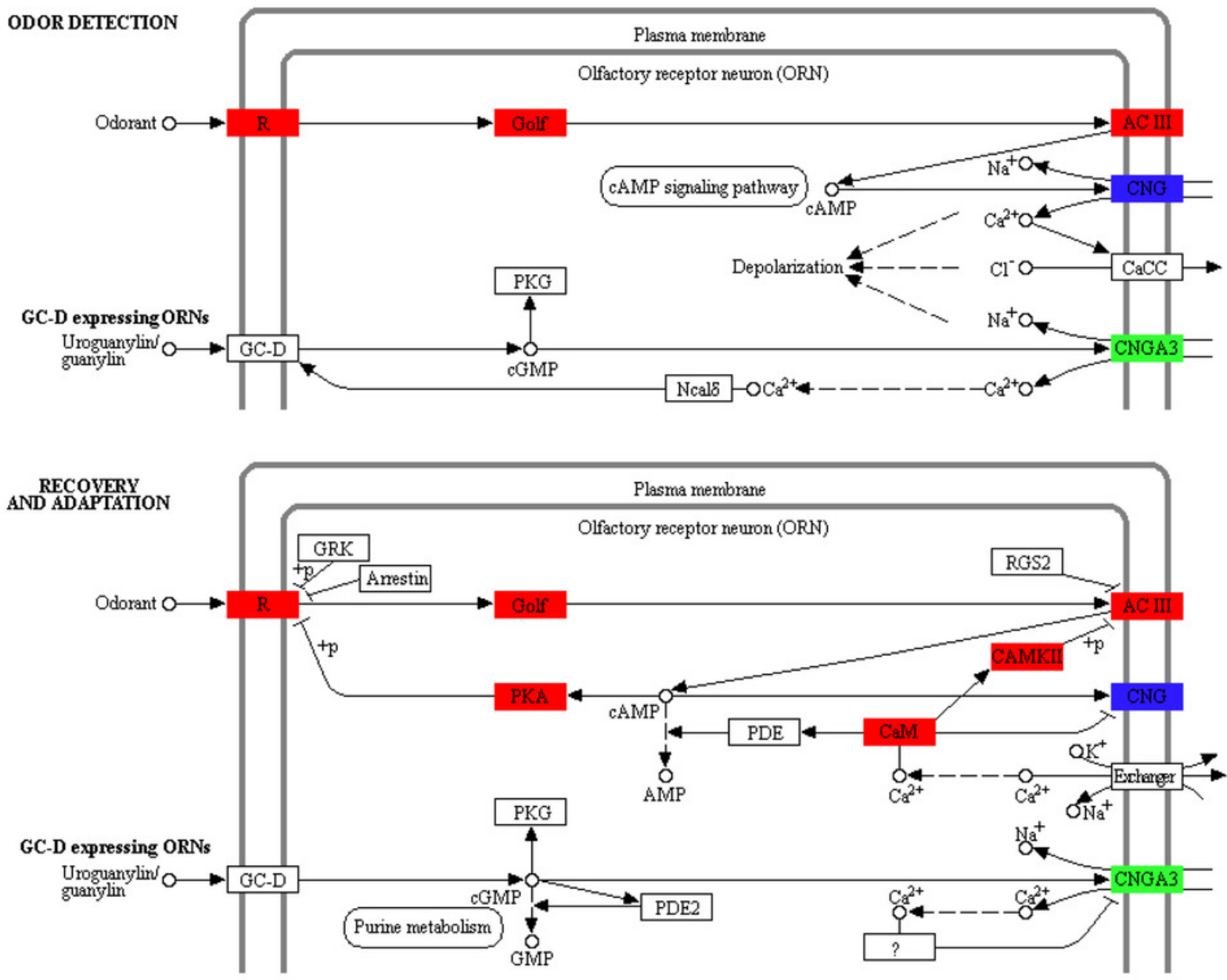
Figure 5

Relative expression levels of nine DEGs related to olfactory transduction.

The results were calculated according to the $2^{-\mathrm{DACT}}$ method using $\beta$-actin as an internal reference gene. ${ }^{*} p<0.05$ and ${ }^{* *} p<0.01$.

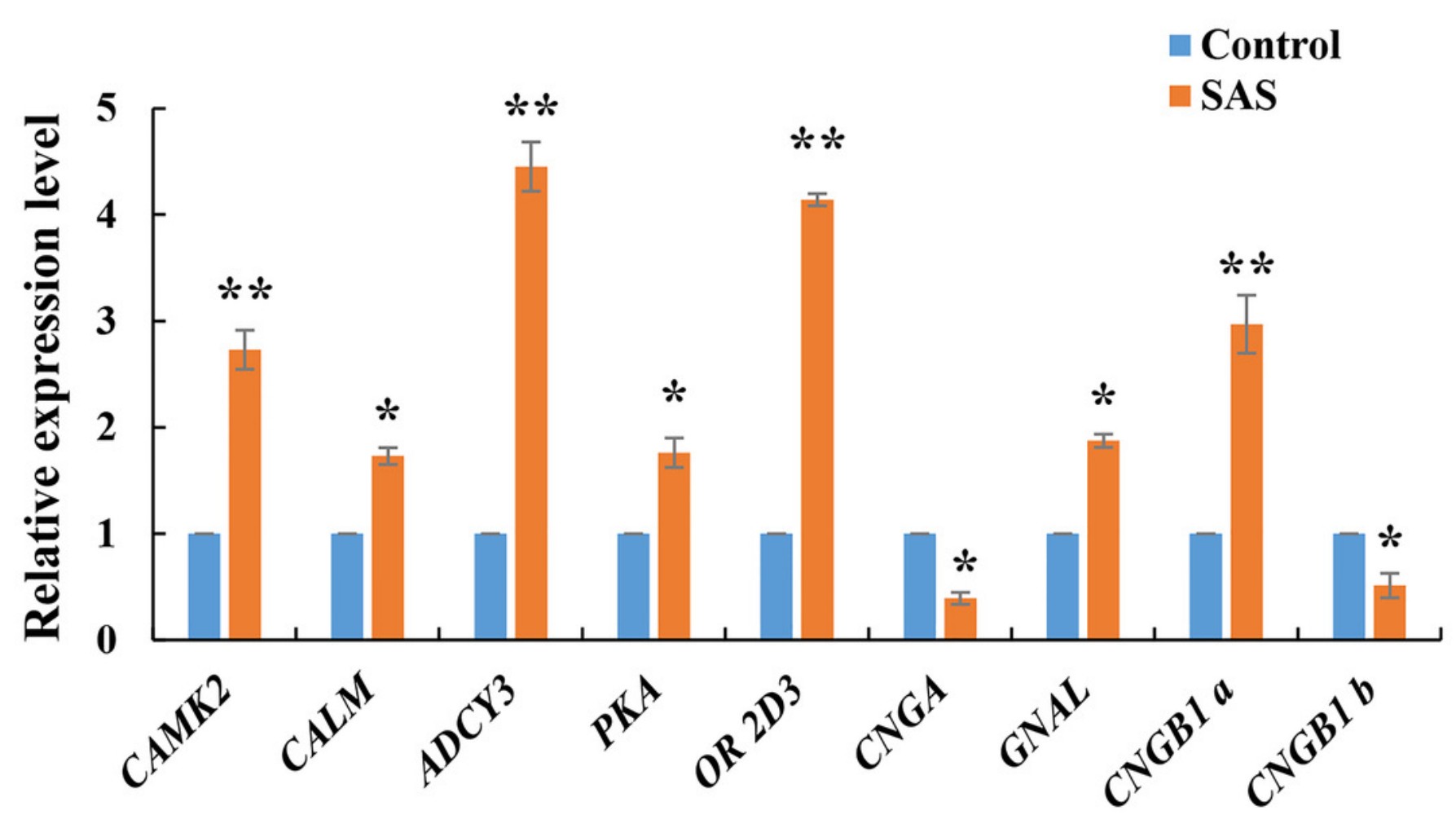




\section{Table $\mathbf{1}$ (on next page)}

Primers for real-time quantitative PCR (RT-qPCR). 
Table 1 Primers for real-time quantitative PCR (RT-qPCR)

\begin{tabular}{|c|c|c|}
\hline Gene name & Gene ID & Primer sequence $\left(5^{\prime} \rightarrow 3^{\prime}\right)$ \\
\hline \multirow[t]{2}{*}{ OR $2 D 3$} & \multirow[t]{2}{*}{ gi|734643370| } & F: CTATGCCAGCACTCTCTTTC \\
\hline & & R: ACAAGGTGGAGGTGAGAA \\
\hline \multirow{2}{*}{$C A L M$} & \multirow{2}{*}{ gi $|698455748|$} & F: AGGGTGTTCATTGGTGCTCG \\
\hline & & R: ATGTAAAGCCCACGACTCAA \\
\hline \multirow{2}{*}{$A D C Y 3$} & \multirow{2}{*}{ gi|734633255| } & F: AACCCATCGTTTCCTAATCC \\
\hline & & R: GCCGCTCTGTTTCTCCTTCT \\
\hline \multirow{2}{*}{$G N A L$} & \multirow{2}{*}{ gi|734649985| } & F: AGCATCGCTCCGCTTTC \\
\hline & & R: ATCCCGCTGACCTCCTACA \\
\hline \multirow{2}{*}{$C A M K 2$} & \multirow{2}{*}{ gi|734594146| } & F: AATGCCACCAACGACGAG \\
\hline & & R: TCCACCAGGTTTCCCAGA \\
\hline \multirow{2}{*}{$C N G A$} & \multirow{2}{*}{ gi $|734644355|$} & F: AAGTGTTTAGCCCTGGAGATTAC \\
\hline & & R: CCGCTTTACTGCCCTTGATA \\
\hline \multirow{2}{*}{$P K A$} & \multirow{2}{*}{ gi|734635100| } & F: AACCCATCGTTTCCTAATCC \\
\hline & & R: GCCGCTCTGTTTCTCCTTCT \\
\hline \multirow{2}{*}{$C N G B 1$ a } & \multirow{2}{*}{ gi|734611524| } & F: GTGTACGACGTAGCCACGAT \\
\hline & & R: TGAGATTCCACTGAGCGATT \\
\hline \multirow{2}{*}{$C N G B 1 b$} & \multirow{2}{*}{ gi|554826775| } & F: ACTTTGTTGGTGTCTTTGCTTT \\
\hline & & R: TCTCGGGGGATGTTGTAGG \\
\hline
\end{tabular}

2 


\section{Table 2(on next page)}

The sequence quality and mapping results between the SAS and Control groups 
Table 2 The sequence quality and mapping results between the SAS and Control groups

\begin{tabular}{lll}
\hline Library & Control & SAS \\
\hline Raw Reads Number: & $39,116,990$ & $39,805,502$ \\
Raw Reads Length (bp): & 125 & 125 \\
Clean Reads Number: & $32,205,388$ & $32,272,020$ \\
Clean Reads Length (bp): & 125 & 125 \\
Clean Reads Rate (\%): & 82.33 & 81.07 \\
Mapped Reads: & $25,684,902$ & $25,830,011$ \\
Mapping Rate(\%): & 80 & 80 \\
Raw Q30 Bases Rate (\%): & 90.57 & 90.23 \\
Clean Q30 Bases Rate (\%): & 95.83 & 96.02 \\
\hline
\end{tabular}




\section{Table 3(on next page)}

KEGG pathway analysis of the 20 highly enriched categories 


\begin{tabular}{lll}
\hline Pathway ID & q-value & Pathway \\
\hline map03010 & $3.77 \mathrm{E}-15$ & Ribosome \\
map05012 & $1.59 \mathrm{E}-08$ & Parkinson's disease \\
map03008 & $1.01 \mathrm{E}-07$ & Ribosome biogenesis in eukaryotes \\
map03040 & $3.40 \mathrm{E}-07$ & Spliceosome \\
map03050 & $1.29 \mathrm{E}-06$ & Proteasome \\
map03013 & $1.72 \mathrm{E}-06$ & RNA transport \\
map00190 & $1.72 \mathrm{E}-06$ & Oxidative phosphorylation \\
map03030 & $1.94 \mathrm{E}-06$ & DNA replication \\
map03430 & $4.59 \mathrm{E}-05$ & Mismatch repair \\
map00970 & 0.000145129 & Aminoacyl-tRNA biosynthesis \\
map05016 & 0.001811227 & Huntington's disease \\
map04740 & 0.004459478 & Olfactory transduction \\
map03440 & 0.005177196 & Homologous recombination \\
map03420 & 0.005828765 & Nucleotide excision repair \\
map04721 & 0.008139829 & Synaptic vesicle cycle \\
map04920 & 0.029436551 & Adipocytokine signaling pathway \\
map04142 & 0.037198669 & Lysosome \\
map05160 & 0.037198669 & Hepatitis C \\
map04111 & 0.041314855 & Cell cycle - yeast \\
\hline
\end{tabular}

2 


\section{Table 4 (on next page)}

Nine highly differentially expressed genes in 8 categories from olfactory transduction 
1 Table 4 nine highly differentially expressed genes in 8 categories from olfactory transduction

\begin{tabular}{|c|c|c|c|c|}
\hline Gene name & $\log _{2}$ FoldChange & P-value & Gene ID & Description \\
\hline OR $2 D 3$ & 5.388277756 & $1.13 \mathrm{E}-11$ & gi $|734643370|$ & Olfactory receptor 2D3 \\
\hline CALM & 2.148730614 & $1.35 \mathrm{E}-25$ & gi|698455748| & Calmodulin \\
\hline$A D C Y 3$ & 4.803315255 & $4.82 \mathrm{E}-05$ & gi $|734633255|$ & Adenylate cyclase type 3 \\
\hline$G N A L$ & 1.303754743 & $9.90 \mathrm{E}-96$ & gi $|734649985|$ & Guanine nucleotide-binding protein $\mathrm{G}(\mathrm{olf})$ subunit alpha \\
\hline$C A M K 2$ & 3.964251474 & $6.05 \mathrm{E}-76$ & gi|734594146| & $\begin{array}{l}\text { Calcium/calmodulin-dependent protein kinase type II subunit } \\
\text { gamma }\end{array}$ \\
\hline$C N G A$ & -1.827161905 & $3.96 \mathrm{E}-06$ & gi|734644355| & $\begin{array}{l}\text { Cyclic nucleotide-gated channel cone photoreceptor subunit } \\
\text { alpha }\end{array}$ \\
\hline$P K A$ & 2.004522347 & $1.89 \mathrm{E}-19$ & gi|734635100| & cAMP-dependent protein kinase catalytic subunit PRKX \\
\hline$C N G B 1 a$ & 4.165885335 & $1.29 \mathrm{E}-47$ & gi|734611524| & Cyclic nucleotide-gated cation channel beta- 1 \\
\hline$C N G B 1 b$ & -4.004039667 & 0.001710637 & gi $|554826775|$ & Cyclic nucleotide-gated cation channel beta-1 \\
\hline
\end{tabular}

